\title{
Implementation of parallel three-point block codes for solving large systems of ordinary differential equations.
}

\begin{abstract}
The three-point fully implicit block methods are developed for solving large systems of ordinary differential equations using variable step size on a parallel shared memory computer. The methods calculate the numerical solution at three points simultaneously and are suitable for parallelization across the method. The methods are in a simple form as Adams Moulton method with the specific aim of gaining efficiency. For large problems, the parallel implementation produced a good speed-up with respect to the sequential timing and hence better efficiency for the methods developed.
\end{abstract}

Keyword: Numerical method; Parallel method; Block method; Three-point block. 